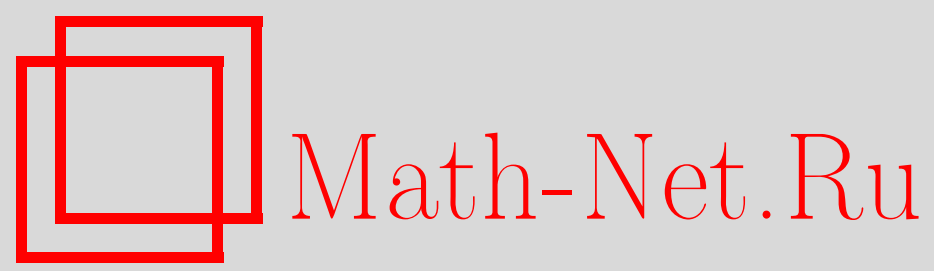

Н. Ю. Мутовкина, Н. А. Семенов, Человекоразумные системы и особенности их функционирования, Нечеткие системы и мягкие вычисления, 2017, том 12 , выпуск 2, 121-131

DOI: https://doi.org/10.26456/fssc28

Использование Общероссийского математического портала Math-Net.Ru подразумевает, что вы прочитали и согласны с пользовательским соглашением

http: //www.mathnet.ru/rus/agreement

Параметры загрузки:

IP : 3.89 .197 .203

26 апреля 2023 г., 12:06:30 


\section{УДК 004.82:510.6 \\ ЧЕЛОВЕКОРАЗУМНЫЕ СИСТЕМЫ И ОСОБЕННОСТИ ИХ ФУНКЦИОНИРОВАНИЯ ${ }^{1}$}

Мутовкина Н.Ю., Семенов Н.А.

Тверской государственный технический университет, г. Тверь

Поступила 17.09.2017, после переработки 12.12.2017.

В работе раскрыта сущность человекоразумных систем, рассмотрены их состав и структура. Основой функционирования человекоразумной системы является антропоцентрический подход. В человекоразумных системах люди, системы поддержки принятия решений и экспертные системы органично дополняют друг друга, используя очевидные преимущества. Люди поддерживают человекоразумную систему в целевом состоянии, являются ведущим звеном системы, организуя ее работу, формулируя и решая частные задачи. Программные компоненты человекоразумной системы выполняют подкрепляющую и советующую функции. Описана проблематика принятия решений в человекоразумных системах, обусловленная субъективностью и неоднозначностью деятельности участников системы. Изучены понятия расплывчатых целей, ограничений и решений в человекоразумных системах, сделана попытка развития общей теории итерационных процессов взаимодействия и принятия решений, которые в разной степени включают расплывчатость и неопределенность. Приведен числовой пример итерационного процесса взаимодействия в абстрактной человекоразумной системе. Рассмотрена задача выявления управляющих воздействий, позволяющих быстрее найти лучшее решение креативной проблемы.

Ключевые слова: человекоразумная система, множество, состояние, управляющее воздействие, принятие решений, неопределенность, расплывчатость.

Нечеткие системы и мягкие вычисления. 2017. Т. 12, № 2. С. 121-131. https://doi.org/10.26456/fssc28

\section{Введение}

Человекоразумные системы (ЧРС) в своем функционировании характеризуются расплывчатостью и неопределенностью, причем эти особенности касаются не только поведения участников системы, но и задаваемых ими целей, задач и выбора инструментов их достижения и решения. В ЧРС решения принимаются в расплывчатых условиях, а принятие решения - процесс итерационный. Прежде, чем перейти к описанию проблематики принятия решений в ЧРС, обусловленной субъективностью и неоднозначностью деятельности участников системы, а

${ }^{1}$ Работа выполнена при финансовой поддержке РФФИ (проект № 15-07-05617-а). 
также выработке рекомендаций по преодолению этих особенностей, следует рассмотреть само понятие «человекоразумная система».

Термин «человекоразумная система» был впервые введен в научный оборот в 2014 году профессором, д.т.н. Кузнецовым В.Н. Как указано в [1], ЧРС представляет собой симбиоз ученых, информационных и программных систем поддержки принятия решений и экспертной системы. В ЧРС осуществляется информационная поддержка решений нетривиальных проблем на основе ориентированной на развитие разума лиц и групп, принимающих решения, петли обратной связи (информация $\rightarrow$ разум $\rightarrow$ решение проблемы $\rightarrow$ информация). При этом применяются методы искусственного интеллекта, методы и эвристические процедуры формирования творческих и интуитивных решений, методы их статистической нечеткой оценки и согласованной оптимизации в условиях расплывчатой неопределенности. ЧРС отличается от «человекоподобной» системы в первую очередь тем, что в своей основе содержит человека-исследователя; человека, высокий уровень интеллекта, а также хорошо развитые гуманистические и лидерские качества которого позволяют ему критически подходить к оценке возможных решений сложных проблем современности. Как неоднократно доказала практика, за весь тот период, что люди считаются «хозяевами» Земли, венцом, так сказать, творения, ими совершено множество ошибок, принято огромное количество неверных решений, некоторые из которых просто фатальны. Причем, бо́льшая часть негативных последствий от неверно принятых решений пришлась именно на XX век и продолжает увеличиваться. Этому способствует необдуманность использования человеком результатов научно-технического прогресса, что характерно для всех областей жизни общества. Для сведения к минимуму негативных последствий принятия людьми неверных решений авторами исследования предлагается разрабатывать и внедрять в разные сферы жизни и деятельности общества ЧРС. Эти системы настроены на выбор такого плана действий по решению сложной проблемы, который максимизирует значение целевой функции общей полезности, но и не допускает фатальной минимизации функции индивидуальной полезности, т.е. ЧРС действует, руководствуясь методами согласованной оптимизации $[2,3]$.

\section{1. Состав и структура человекоразумных систем}

В ЧРС входят как управляющие, так и управляемые элементы.

Управляющие элементы - люди (ученые, исследователи, эксперты, лица, принимающие решения), которые обладают достаточными знаниями и компетентностью в смежных предметных областях, что позволяет им, досконально разобравшись в ситуации, предложить такое решение проблемы, которое устроит всех участников процесса принятия решения примерно в равной степени, уж точно не меньше, чем минимально допустимое значение целевой функции каждого из них. При этом всегда существует вероятность несовпадения целей и интересов управляющих элементов, а это оказывает негативное воздействие на управляемые элементы, которые, под натиском разнородных данных входят в состояние коллапса и начинают выдавать нелогичные, зачастую диаметрально противоположные результаты. Поэтому актуальным представляется применение методов и моделей недопущения и решения конфликтных ситуаций [4] в ЧРС и, прежде всего, в ее управляющей части. Управляющие элементы в дальнейшем будут обозначаться как элементы вида А. 
Управляемые элементы в ЧРС разделяются на два вида: В и С. К виду В относятся системы поддержки принятия решений (СППР) и экспертные системы $(Э \mathrm{C})$, обладающие признаками интеллектуальности. Эти системы моделируют поведение эксперта в конкретной предметной области. Однако современные проблемы настолько сложны, что для их решения требуются знания из нескольких предметных областей, поэтому в ЧРС предусмотрен междисциплинарный подход к анализу проблемной области. Элементы из группы В обеспечивают:

1. информацию о текущем состоянии проблемы, анализ сообщений и привлечение внимания ЛПР к каким-либо изменениям в проблемной ситуации;

2. интерактивное проведение диагностики причин изменений;

3. выбор необходимых действий по устранению причин нежелательных изменений (проблема не должна усугубляться) [5].

ЛПР как получает сведения от элементов вида В, так и закладывает в них факты и правила. И здесь на первое место в иерархии важностей выступает «разумность» ЛПР, обусловленная не только уровнем его интеллекта, но и сформированными с его рождения человеческими качествами. Любое искажение фактов и правил, в т.ч. в угоду личной выгоде, недопустимо, потому как это может повлечь необратимые последствия, которые обернутся против самого человека, «подтасовавшего» факты. ЛПР сообщает свои оценки через специальный интерфейс программу, обеспечивающую взаимодействие ЛПР с ЭС и/или СППР. Совокупность правил, которые позволяют получать ответы на запросы со стороны интерфейса, называется механизмом логического вывода (МЛВ). МЛВ обращается за необходимыми данными к базе знаний (БЗ), представляющей собой набор фактов и некоторых правил, которые не входят в МЛВ. Особенностью БЗ является то, что ее структура (в отличие от структуры обычных баз данных) позволяет вести поиск оптимального решения «разумно», исключая заведомо ложные ветви рассуждений.

Группу C составляют пассивные элементы, не имеющие целей и каких-либо предпочтений, мнений, а выполняющие роль вспомогательных инструментов для элементов вида А и в некоторой степени - вида В (Рис. 1).

$\mathrm{B}$ частности, элементами вида $\mathrm{C}$ являются программные продукты, позволяющие работать с нечетко определенными числовыми величинами, которые могут выражаться в виде распределения нечетких мер, позволяющих более достоверно описывать реальные явления и процессы, требующие незамедлительной реакции человека.

На Рис. 1 показано, что элементы видов А и В обладают множеством целей и возможностью возникновения конфликтных ситуаций из-за расхождений в целях и подходах к решению одной и той же проблемы. Элементы вида $\mathrm{C}$ могут конфликтовать друг с другом вследствие несогласованности совместной деятельности элементов вида А и В.

В ЧРС возникают качественно новые свойства поведения управляющих элементов, которые, в отличие от обычного, среднестатистического человека (к сожалению, не каждый человек разумен в прямом смысле этого слова) всегда действуют рационально, в соответствии с законами логики и, не забывая при этом о гуманистических ценностях.

Для ЧРС характерно понятие «полезности» (utility). Полезностью в ЧРС называется потенциальная доступность как управляющих, так и управляемых элементов, с которыми любой из элементов может связаться в случае необходимости 


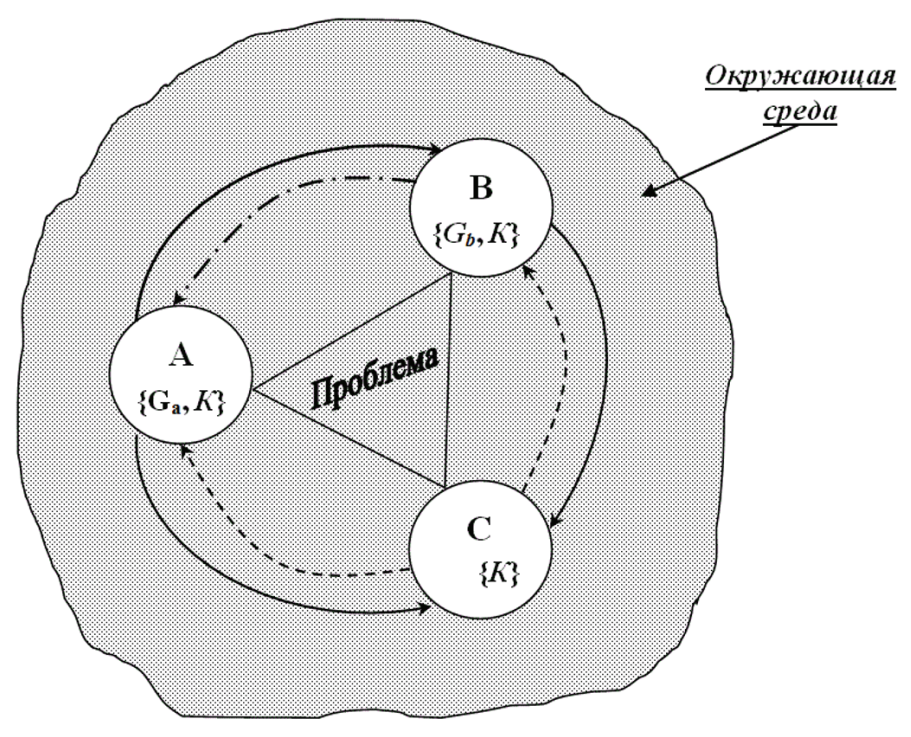

Рис. 1: Состав человекоразумной системы

и при этом каждый из них получит некоторый положительный эффект (приращение собственной функции полезности).

\section{2. Итерационные процессы взаимодействия управляющих и управляе- мых элементов в ЧРС}

Взаимодействие в ЧРС между управляющими и управляемыми элементами осуществляется с целью решения поставленной сложной задачи и представляет собой итерационный процесс. При этом наибольший интерес в силу сложности и неоднозначности поведения представляет взаимодействие элементов видов А и B.

Пусть управляемый элемент $B_{j}, j=\overline{1, m}$ имеет конечное число состояний и под воздействием со стороны управляющего элемента $A_{i}, i=\overline{1, n}$, может переходить из одного состояния в другое. Каждое состояние $s_{t}$, в котором элемент $B_{j}$ находится в момент времени $t, t=0,1,2, \ldots$, принадлежит заданному конечному множеству возможных состояний $S=\left\{s_{1}, \ldots, s_{p}\right\}$; при этом имеющее место в момент $t$ управляющее воздействие $u(t)$ является элементом множества $U=\left\{\alpha_{1}, \ldots, \alpha_{g}\right\}$. Динамика элемента $B_{j}$ во времени описывается уравнением состояния:

$$
s_{t+1}=f\left(s_{t}, u_{t}\right), \quad t=0,1,2, \ldots,
$$

в котором $f$ - заданная функция, отображающая $S \times U$ в $S$. Так что $f\left(s_{t}, u_{t}\right)$ представляет собой следующее состояние для $s_{t}$ при управляющем воздействии $u_{t}$. В силу субъективности предпочтений и взглядов участников вида А на решение одной и той же проблемы функция $f$ является случайной. Тогда состояние элемента $B_{j}$ в момент $t+1$ характеризуется распределением вероятностей $P\left(s_{t+1} \mid s_{t}, u_{t}\right)$ на $S$, условным по $s_{t}$ и $u_{t}$. Предполагается, что в каждый момент времени $t$ на поведение управляющего элемента по отношению к элементу $B_{j}$ налагается расплывчатое ограничение $\tilde{Z}_{t}$, являющееся расплывчатым множеством 
в $U$ с функцией принадлежности $\mu_{t}\left(u_{t}\right)$. Ограничения налагаются в соответствии с логикой рассуждений элемента $A_{i}$, не противоречащей здравому смыслу. Цель представляет собой расплывчатое множество $\tilde{Q}_{T}$ в $S$, определяемое функцией принадлежности $\mu_{\tilde{Q}_{T}}\left(s_{T}\right)$, где $T-$ окончание периода, отведенного на решение задачи. Предполагается, что в момент $T+1$ решение задачи уже будет не актуально. В начале взаимодействия элемент $B_{j}$ находится в начальном состоянии $s_{0}$. Функционирование элемента $B_{j}$ определяется условным распределением вероятностей $P\left(s_{t+1} \mid s_{t}, u_{t}\right)$. Элемент $A_{i}$ должен максимизировать вероятность достижения расплывчатой цели $\tilde{Q}_{T}$ к моменту $T$ при условии выполнения расплывчатых ограничений $\tilde{Z}_{0}, \ldots, \tilde{Z}_{T-1}$. Если расплывчатая цель $\tilde{Q}_{T}$ рассматривается как расплывчатое событие [6] в пространстве $S$, то условная вероятность этого события при фиксированных $s_{T-1}$ и $u_{T-1}$ рассчитывается как условное математическое ожидание:

$$
M_{\mu_{Q_{T}}}\left(s_{T}\right)=\sum_{s_{T}} p\left(s_{T} \mid s_{T-1}, u_{T-1}\right) \cdot \mu_{Q_{T}}\left(s_{T}\right),
$$

где $\mu_{Q_{T}}-$ функция принадлежности расплывчатой цели.

Задача сводится к нахождению стратегии, состоящей из последовательности управляющих воздействий $u_{0}, \ldots, u_{T-1}$, максимизирующей значение целевой функции. Итерационность процесса позволяет представить решение в виде:

$$
u_{t}=v_{t}\left(s_{t}\right), \quad t=0,1,2, \ldots,
$$

где $v_{t}$ - принятая стратегия выбора управляющего воздействия $u_{t}$ в зависимости от реализовавшегося $s_{t}$. После этого для получения как $v_{t}$, так и максимизирующего решения $u_{0}^{*}, \ldots, u_{T-1}^{*}$ можно применить метод динамического программирования. Для реализации метода предлагается применить рекуррентные соотношения (4), наподобие тех, что представлены в [7]:

$$
\begin{gathered}
\mu_{Q_{T-\tau}}\left(s_{T-\tau}\right)=\max _{u_{T-\tau}}\left(\mu_{T-\tau}\left(u_{T-\tau}\right), M \mu_{Q_{T-\tau+1}}\left(s_{T-\tau+1}\right)\right), \\
\quad=\mu_{Q_{T-\tau+1}}\left(s_{T-\tau+1}\right)= \\
\sum_{s_{T-\tau+1}} P\left(s_{T-\tau+1} \mid s_{T-\tau}, u_{T-\tau}\right) \mu_{Q_{T-\tau+1}}\left(s_{T-\tau+1}\right),
\end{gathered}
$$

где $\mu_{Q_{T-\tau}}\left(s_{T-\tau}\right)-$ функция принадлежности расплывчатой цели в момент $t=T-\tau$, индуцированной расплывчатой целью в момент $t=T-\tau+1, \tau=\overline{1, T}$.

Например, пусть интеллектуальная ЭС имеет 5 состояний: «не соответствует цели» $\left(s_{1}\right)$, «незначительно соответствует цели» $\left(s_{2}\right)$, «соответствует цели примерно на $50 \% »\left(s_{3}\right)$, «может обеспечить достижение цели, но для этого требуется некоторое расширение БЗ» $\left(s_{4}\right)$, «соответствует цели на 100\%» $\left(s_{5}\right)$. Состояние $s_{1}$ указывает на то, что ЭС «не понимает» поставленную цель, поскольку использует факты и правила без обновления информации о новой задаче. Состояние $s_{2}$ означает, что ЭС не может обеспечить эффективную поддержку элементу $A_{1}$, поскольку не обладает достаточной информацией о проблемной области, однако эту ситуацию можно исправить, дополнив БЗ системы необходимой информацией. Состояния $s_{3}$ и $s_{4}$ также требуют дополнений и изменений БЗ системы, но в разных объемах. Состояние $s_{5}$ является наиболее эффективным состоянием ЭС, позволяющим $A_{1}$ скорее достичь поставленную цель. Проблема заключается в том, что $A_{1}$ должен найти такие управляющие воздействия (последовательности обновлений и дополнений БЗ ЭС), чтобы текущее состояние системы не изменилось на худшее и система достигла состояния $s_{5}$ за конечное число итераций. 
При этом время для решения поставленной задачи у $A_{1}$ ограничено, а это предполагает его взаимодействие с ӘС независимо от ее состояния в момент $t$. Пусть $A_{1}$ обладает знаниями о проблемной области, которыми не обладает система. Его управляющие воздействия сводятся к введению в систему этих знаний в определенной последовательности с учетом изменений и дополнений в рассматриваемых предметной и проблемной областях. Эти изменения и дополнения обозначаются входными сигналами $\alpha_{1}, \alpha_{2}$ и $\alpha_{3}$, т.е. $g=3$, а распределение вероятностей $P\left(s_{t+1} \mid s_{t}, u_{t}\right)$ задается Таблицей 1 . Время $T=2$.

Таблица 1: Распределение вероятностей

\begin{tabular}{|c|c|c|c|c|c|}
\hline \multicolumn{6}{|c|}{$u_{t}=\alpha_{1}$} \\
\hline $\begin{array}{l}s_{t+1} \\
s_{t}\end{array}$ & $s_{1}$ & $s_{2}$ & $s_{3}$ & $s_{4}$ & $s_{5}$ \\
\hline$s_{1}$ & 0.5 & 0.15 & 0.1 & 0.1 & 0.15 \\
\hline$s_{2}$ & 0.3 & 0.1 & 0.1 & 0.2 & 0.3 \\
\hline$s_{3}$ & 0.7 & 0 & 0 & 0.2 & 0.1 \\
\hline$s_{4}$ & 0 & 0.3 & 0.2 & 0 & 0.5 \\
\hline$s_{5}$ & 0.4 & 0.1 & 0.25 & 0.15 & 0.1 \\
\hline \multicolumn{6}{|c|}{$u_{t}=\alpha_{2}$} \\
\hline$s_{1}$ & 0.1 & 0.3 & 0.3 & 0.2 & 0.1 \\
\hline$s_{2}$ & 0.3 & 0.3 & 0 & 0.3 & 0.1 \\
\hline$s_{3}$ & 0.4 & 0.3 & 0.3 & 0 & 0 \\
\hline$s_{4}$ & 0.2 & 0.2 & 0.5 & 0 & 0.1 \\
\hline$s_{5}$ & 0.4 & 0.4 & 0 & 0 & 0.2 \\
\hline \multicolumn{6}{|c|}{$u_{t}=\alpha_{3}$} \\
\hline$s_{1}$ & 0.3 & 0.1 & 0.1 & 0 & 0.5 \\
\hline$s_{2}$ & 0.25 & 0.25 & 0 & 0.4 & 0.1 \\
\hline$s_{3}$ & 0.8 & 0 & 0 & 0.1 & 0.1 \\
\hline$s_{4}$ & 0.4 & 0.5 & 0.1 & 0 & 0 \\
\hline$s_{5}$ & 0.2 & 0.3 & 0.4 & 0.1 & 0 \\
\hline
\end{tabular}

Например, число 0.2 в положении $\left(s_{1}, s_{3}\right)$ при $u_{t}=\alpha_{1}$ означает, что если в момент времени $t$ ӘС находится в состоянии $s_{1}$ и $A_{1}$ оказывает воздействие $\alpha_{1}$, то в момент $t+1$ система с вероятностью 0.2 окажется в состоянии $s_{3}$.

Пусть расплывчатая цель в момент времени $t=2$ определяется функцией: $\mu_{Q_{2}}\left(s_{1}\right)=0.2, \mu_{Q_{2}}\left(s_{2}\right)=0.35, \mu_{Q_{2}}\left(s_{3}\right)=0.5, \mu_{Q_{2}}\left(s_{4}\right)=0.75, \mu_{Q_{2}}\left(s_{5}\right)=1$, а расплывчатые ограничения в моменты $t=0$ и $t=1$ задаются функциями: $\mu_{0}\left(\alpha_{1}\right)=0.5, \mu_{0}\left(\alpha_{2}\right)=0.7, \mu_{0}\left(\alpha_{3}\right)=0.9, \mu_{1}\left(\alpha_{1}\right)=0.9, \mu_{1}\left(\alpha_{2}\right)=1, \mu_{1}\left(\alpha_{3}\right)=0.8$. Применяя (4), найдем $M \mu_{Q_{2}}\left(s_{k}\right)$ как функцию от $s_{1}$ и $u_{1}$ (Таблица 2). Следовательно, в дальнейших расчетах последовательно участвуют $\mu_{1}\left(\alpha_{3}\right)=0.8$, $\mu_{1}\left(\alpha_{1}\right)=0.9, \mu_{1}\left(\alpha_{1}\right)=0.9, \mu_{1}\left(\alpha_{1}\right)=0.9$ и $\mu_{1}\left(\alpha_{1}\right)=0.9$ при $t=1$.

Далее, руководствуясь (4) при $\tau=1$ и вычисляя $\mu_{Q_{1}}\left(s_{k}\right)$, находим: $\mu_{Q_{1}}\left(s_{1}\right)=0.65, \mu_{Q_{1}}\left(s_{2}\right)=0.60, \mu_{Q_{1}}\left(s_{3}\right)=0.39, \mu_{Q_{1}}\left(s_{4}\right)=0.71$ и $\mu_{Q_{1}}\left(s_{5}\right)=0.45$. Соответствующая оптимальная стратегия имеет вид:

$$
v_{1}\left(s_{1}\right)=\alpha_{3}, \quad v_{1}\left(s_{2}\right)=\alpha_{1}, \quad v_{1}\left(s_{3}\right)=\alpha_{1}, \quad v_{1}\left(s_{4}\right)=\alpha_{1}, \quad v_{1}\left(s_{5}\right)=\alpha_{1}
$$

Результаты последней итерации для $\tau=2$ приведены в Таблице 3. 
Таблица 2: Значения условного математического ожидания $M_{Q_{2}}\left(s_{k}\right)$

\begin{tabular}{|l|l|l|l|l|l|}
\hline $\begin{array}{l}s_{k} \\
u_{1}\end{array}$ & $s_{1}$ & $s_{2}$ & $s_{3}$ & $s_{4}$ & $s_{5}$ \\
\hline$\alpha_{1}$ & 0.43 & 0.60 & 0.39 & 0.71 & 0.45 \\
\hline$\alpha_{2}$ & 0.53 & 0.49 & 0.34 & 0.46 & 0.42 \\
\hline$\alpha_{3}$ & 0.65 & 0.54 & 0.34 & 0.31 & 0.42 \\
\hline
\end{tabular}

Таблица З: Значения условного математического ожидания $M \mu_{Q_{1}}\left(s_{k}\right)$

\begin{tabular}{|l|l|l|l|l|l|}
\hline$s_{k}$ & $s_{1}$ & $s_{2}$ & $s_{3}$ & $s_{4}$ & $s_{5}$ \\
$u_{1}$ & & & & & \\
\hline$\alpha_{1}$ & 0.59 & 0.57 & 0.64 & 0.48 & 0.57 \\
\hline$\alpha_{2}$ & 0.55 & 0.63 & 0.56 & 0.49 & 0.59 \\
\hline$\alpha_{3}$ & 0.52 & 0.64 & 0.64 & 0.60 & 0.54 \\
\hline
\end{tabular}

Исходя из максимальных значений в столбцах табл. 3, очевидно, что в дальнейших расчетах последовательно участвуют $\mu_{0}\left(\alpha_{1}\right)=0.5, \mu_{0}\left(\alpha_{3}\right)=0.9$, $\mu_{0}\left(\alpha_{1}\right)=0.5$ или $\mu_{0}\left(\alpha_{3}\right)=0.9, \mu_{0}\left(\alpha_{3}\right)=0.9$ и $\mu_{0}\left(\alpha_{2}\right)=0.7$ при $t=0$.

По аналогии с предыдущей итерацией получаем: $\mu_{Q_{0}}\left(s_{1}\right)=0.5$, $\mu_{Q_{0}}\left(s_{2}\right)=0.64, \mu_{Q_{0}}\left(s_{3}\right)=0.5, \mu_{Q_{0}}\left(s_{4}\right)=0.6$ и $\mu_{Q_{0}}\left(s_{5}\right)=0.59$. Соответствующая оптимальная стратегия имеет вид:

$$
v_{0}\left(s_{1}\right)=\alpha_{1}, \quad v_{0}\left(s_{2}\right)=\alpha_{3}, \quad v_{0}\left(s_{3}\right)=\alpha_{1}, \quad v_{0}\left(s_{4}\right)=\alpha_{3}, \quad v_{0}\left(s_{5}\right)=\alpha_{2} .
$$

Значения функции $\mu_{Q_{0}}\left(s_{k}\right)$ при $\tau=2$ представляют собой вероятности достижения поставленной цели в момент $t=2$ в предположении, что взаимодействие начинается с состояний $s_{1}, s_{2}, s_{3}, s_{4}$ и $s_{5}$ соответственно и что управляющие воздействия выбираются в соответствии с оптимальной стратегией $v_{t}$, которая задается выражениями (5) и (6).

Если взаимодействие типа $\mathrm{A} \rightarrow \mathrm{B}$ сводится к управлению, т.е. оказанию управляющих воздействий на подчиненные элементы для достижения поставленной цели, то взаимодействие типа $\mathrm{A} \leftrightarrow \mathrm{A}$ можно рассматривать как взаимовлияние, которое дает положительный эффект лишь в состояниях сотрудничества и компромисса.

\section{Заключение}

В работе представлен способ описания взаимодействия управляющих и управляемых элементов ЧРС, основанный на теории нечеткой логики. В частности, рассмотрен метод выявления стратегии поведения управляющего элемента по отношению к интеллектуальной ЭС. От того, какие факты и правила внесет в систему управляющий элемент, зависит эффективность ее работы и полезность выдаваемых результатов. В данной ситуации интересным представляется вопрос о согласовании стратегий нескольких управляющих элементов на одну и ту же ЭС. Для этого могут быть адаптированы методы согласования, представленные, например, в [3, 4].

В силу субъективности мнений, суждений и представлений управляющих элементов их цели, ограничения и решения являются расплывчатыми, нечеткими, 
однако суть решаемой задачи всегда понятна каждому участнику ЧРС. В силу разумности управляющих элементов вариация между их представлениями относительно проблемы и путей ее решения минимальна, иначе система уже не может интерпретироваться как человекоразумная. Тем не менее, отклонения, пусть и не существенные, присутствуют всегда. Для устранения подобных противоречий и подведения управляющих элементов к единому мнению хорошим способом представляется построение их моделей поведения во взаимодействии друг с другом на основе теории нечетких множеств и мягких вычислений.

\section{Список литературы}

[1] Семенов Н.А., Кузнецов В.Н., Клюшин А.Ю., Мутовкина Н.Ю. Человекоразумные программные системы интеллектуальной поддержки решений креативных проблем // Программные продукты и системы. 2015. № 3. С. 12-18.

[2] Мутовкина Н.Ю. Методы согласованной оптимизации технического перевооружения промышленных предприятий: дис. ... канд. техн. наук. Тверь: ТвГТУ, 2009. 219 с.

[3] Кузнецов В.Н., Мутовкина Н.Ю., Доропей В.Н. Согласование и оптимизация управленческих решений в финансово-хозяйственных системах: монография. Тверь: Изд-во ТвГТУ, 2014. 160 с.

[4] Мутовкина Н.Ю., Кузнецов В.Н., Клюшин А.Ю. Методы согласованного управления конфликтом в многоагентной системе // Системы управления и информационные технологии. 2014. Т. 57, № 3.2. С. 255-261.

[5] Аксенов К.А., Гончарова Н.В. Моделирование и принятие решений в организационно-технических системах: учебное пособие. Ч. 1. Екатеринбург: Изд-во Уральского университета, 2015. 104 с.

[6] Zadeh L.A. Probability measures of fuzzy events // Journal of Mathematical Analysis and Applications. 1968. Vol. 23, № 2. Pp. 421-427.

[7] Беллман Р., Заде Л. Принятие решений в расплывчатых условиях // Вопросы анализа и процедуры принятия решений. М.: Мир, 1976. С. 172-215.

\section{Образец цитирования}

Мутовкина Н.Ю., Семенов Н.А. Человекоразумные системы и особенности их функционирования // Нечеткие системы и мягкие вычисления. 2017. Т. 12, № 2 . C. 121-131. https://doi.org/10.26456/fssc28

\section{Сведения об авторах}

\section{1. Мутовкина Наталия Юрьевна}

доцент кафедры бухгалтерского учета и финансов Тверского государственного технического университета.

Россия, 170023, г. Тверь, ул. Маршала Конева, д. 12, ТвГТУ.

E-mail: letter-boxNM@yandex.ru 
2. Семенов Николай Александрович

профессор кафедры информационных систем Тверского государственного технического университета.

Россия, 170023, г. Тверь, проспект Ленина, д. 25, ТвГТУ.

E-mail: slt1155@mail.ru 


\title{
HUMAN-INTELLIGENT SYSTEMS AND FEATURES OF THEIR FUNCTIONING
}

\author{
Mutovkina Nataliya Yuryevna \\ Associate Professor at Accounting and Finance department, \\ Tver State Technical University \\ Russia, 170023, Tver, Marshal Konev st., 12, TvSTU. \\ E-mail: letter-boxNM@yandex.ru \\ Semenov Nikolai Alexandrovich \\ Professor at Information Systems department, \\ Tver State Technical University \\ Russia, 170023, Tver, Lenin av., 25, TvSTU. E-mail: slt1155@mail.ru
}

Received 17.09.2017, revised 12.12.2017.

The essence of human-intelligent systems is revealed in the work, their composition and structure are considered. The basis for the functioning of a human-intelligent system is the anthropocentric approach. In humanintelligent systems, people, decision support systems and expert systems organically complement each other, using obvious advantages. People support a human-intelligent system in the target state, they are the leading link in the system, organizing its work, formulating and solving particular problems. The software components of the human-intelligent system perform reinforcing and advisory functions. The problematic of decision-making in human-intelligent systems is described, conditioned by the subjectivity and ambiguity of the activity of participants in the system. The concepts of vague goals, limitations and decisions in human-intelligent systems are studied, an attempt is made to develop a general theory of iterative processes of interaction and decision-making, which to varying degrees include vagueness and uncertainty. A numerical example of the iterative process of interaction in an abstract human-intelligent system is given. The problem of revealing control actions, allowing to find the best solution of the creative problem more quickly, is considered.

Keywords: human-intelligent system, multitude, condition, management influence, decision-making, uncertainty, vagueness.

Nechetkie Sistemy i Myagkie Vychisleniya [Fuzzy Systems and Soft Computing], 2017, vol. 12(2), pp. 121-131. https://doi.org/10.26456/ fssc 28

\section{References}

[1] Semenov N.A., Kuznetsov V.N., Klyushin A.Yu., Mutovkina N.Yu. Humanpurpose software systems for intellectual support of solutions to creative problems. Programmnye Produkty i Sistemy [Software Products and Systems], 2015, no. 3, pp. 12-18. (in Russian) 
[2] Mutovkina N.Yu. Metody Soglasovannoi Optimizatsii Tekhnicheskogo Perevooruzheniya Promyshlennykh Predpriyatii [Methods of Coordinated Optimization of Technical Re-equipment of Industrial Enterprises]. PhD thesis. TSTU Publ., Tver, 2009. 219 p. (in Russian)

[3] Kuznetsov V.N., Mutovkina N.Yu., Doropey V.N. Soglasovanie i Optimizatsiya Upravlencheskikh Reshenii $v$ Finansovo-Khozyaistvennykh Sistemakh: Monografiya [Coordination and Optimization of Management Decisions in Financial and Economic Systems: Monograph]. TSTU Publ., Tver, 2014. 160 p. (in Russian)

[4] Mutovkina N.Yu., Kuznetsov V.N., Klyushin A.Yu. Methods of coordinated conflict management in a multi-agent system. Sistemy Upravleniya $i$ Informatsionnye Tekhnologii [Control Systems and Information Technology], 2014, vol. 57(3.2), pp. 255-261. (in Russian)

[5] Aksenov K.A., Goncharova N.V. Modelirovanie $i$ Prinyatie Reshenii v Organizatsionno-Tekhnicheskikh Sistemakh: Uchebnoe Posobie [Modeling and Decision Making in Organizational and Technical Systems: Textbook]. Vol. 1. Ural University Publ., Ekaterinburg, 2015. 104 p. (in Russian)

[6] Zadeh L.A. Probability Measures of Fuzzy Events. Journal of Mathematical Analysis and Applications, 1968, vol. 23(2), pp. 421-427.

[7] Bellman R., Zadeh L. Decision-making under vague conditions. Voprosy Analiza $i$ Protsedury Prinyatiya Reshenii [Analysis Questions and Decision-Making Procedures]. Mir Publ., Moscow, 1976. Pp. 172-215. (in Russian)

\section{Citation}

Mutovkina N.Yu., Semenov N.A. Human-intelligent systems and features of their functioning. Nechetkie Sistemy i Myagkie Vychisleniya [Fuzzy Systems and Soft Computing], 2017, vol. 12(2), pp. 121-131. (in Russian). https://doi.org/10.26456/ fssc 28 